\title{
Logística Humanitaria: modelos para la atención de poblaciones afectadas por desastres naturales
}

\author{
Esther Berger Vidal ${ }^{1}$, Carmela Velásquez Pino ${ }^{1}$, Caridad Huaroto Sumari ${ }^{1}$, \\ María Zacarías Díaz ${ }^{1}$, Luis Núñez Ramírez ${ }^{1}$ y Justo Arriola Sánchez ${ }^{1}$
}

Resumen: Ante la tendencia creciente del impacto de los desastres naturales, se requiere brindar ayuda inmediata durante un plazo determinado hasta que la población afectada recupere una calidad de vida adecuada. La Logística Humanitaria, es un enfoque para atender a tales poblaciones, a través de una cadena de suministro conformada por entidades públicas y privadas, centralizadas en el Perú por el Instituto Nacional de Defensa Civil, que acopia, envía y distribuye suministros, médicos y rescatistas. El objetivo es optimizar la ayuda para brindarla con eficiencia e inmediatez. Bajo el enfoque de Logística Humanitaria se usaron modelos de simulación, inventarios y localización para definir la gestión óptima de los almacenes de suministros y la localización óptima de refugios y se analizó el riesgo de inundaciones en el distrito de Chosica, caso de estudio. Como resultado se han planteado modelos de simulación de inventarios y de localización generadores de soluciones para escenarios diversos. Se concluye que es posible sincronizar los requerimientos de ayuda con las necesidades en las zonas afectadas manteniendo abastecidos los almacenes en su máxima capacidad a lo largo del tiempo; que con información geológica suficiente es posible, mediante modelos de localización, determinar lugares seguros de refugio y atención.

Palabras clave: logística humanitaria; inventarios; localización..

\section{Humanitarian Logistics: models for the attention of populations affected by natural disasters}

\begin{abstract}
Given the growing trend of the impact of natural disasters, it is necessary to provide immediate help for a certain period of time until the affected population recovers an adequate quality of life. The Humanitarian Logistics, is an approach to serve such populations, through a Supply Chain made up of public and private entities, centralized in Peru by National Institute of Civil Defense, which collects, sends and distributes supplies, doctors and rescuers. The objective is to optimize the help to provide it with efficiency and immediacy. Under the Humanitarian Logistics approach, simulation, inventory and localization models were used to define the optimal management of supply warehouses and the optimal location of refuges and the risk of flooding in the district of Chosica, case study, was analyzed. As a result, simulation models for inventories and localization have been proposed that generate solutions for different scenarios. It is concluded that it is possible to synchronize the aid requirements with the needs in the affected areas keeping the stores supplied at their maximum capacity over time; that with sufficient geological information it is possible, by means of location models, to determine safe places of refuge and attention.
\end{abstract}

Keywords: humanitarian logistics; inventories; location.

Recibido: 24/09/2018. Aceptado: 18/11/2018. Publicado online: 31/12/2018.

(C) Los autores. Este artículo es publicado por la Revista PESQUIMAT de la Facultad de Ciencias Matemáticas, Universidad Nacional Mayor de San Marcos. Este es un artículo de acceso abierto, distribuido bajo los términos de la licencia Creative Commons Atribucion-No Comercia-Compartir Igual 4.0 Internacional. (http://creativecommons.org/licenses/by-nc-sa/4.0/) que permite el uso no comercial, distribución y reproducción en cualquier medio,

${ }^{1}$ UNMSM, Facultad de Ciencias Matemáticas. e-mail: ebergerv@unmsm.edu.pe, cvelasquezp@unmsm.edu.pe, jhuarotos@unmsm.edu.pe, mzacariasd@unmsm.edu.pe, lnunezr@unmsm.edu.pe, justo.arriola@unmsm.edu.pe. 


\section{Introducción}

En el año 2017, el Perú fue afectado por las consecuencias del Fenómeno El Ni?o Costero, que generó gran cantidad de inundaciones en cientos de distritos de la costa del país, entre ellos el distrito de Chosica ubicado en la parte oriental de la provincia de Lima y en la cuenca media del río R?mac que siendo la puerta de entrada desde Lima a los Andes centrales está expuesto al riesgo de deslizamientos de detritos o huaycos, lluvias e inundaciones.

Los daños a nivel nacional, producidos por las inundaciones del año 2017, reportados en [6], indicaron que el impacto económico fue equivalente al 1,7\% del PBI, 874 distritos fueron declarados en emergencia, hubo 184 mil damnificados, un millón de afectados, 21 mil viviendas colapsaron, 20 mil quedaron inhabitables, 710 establecimientos de salud fueron afectados, 39 establecimientos de salud colapsaron, 3 mil kilómetros de la red nacional vial fueron afectados, 323 puentes quedaron destruidos, 25 mil hectáreas de cultivo se perdieron, 60 mil hectáreas de cultivo fueron afectadas, 113 personas murieron, 17 desaparecieron y se reportaron hasta esa fecha 397 heridos.

Esa problemática fue la motivación para estudiar este problema tomando como caso el distrito de Chosica que fue uno de los altamente afectados durante ese evento, pero que además sufre anualmente el deslizamiento de detritos identificados como huaycos e inundaciones, que se producen durante el verano costero. Existe una problemática asociada a la falta de planificación urbana y a la ubicación arbitrariamente elegida por los pobladores quienes suelen construir sus viviendas a orillas de los ríos Rímac y Huaycoloro o en las laderas y quebradas de los cerros por donde existe el peligro que discurra el agua en las épocas de lluvias intensas. Así, Chosica contribuye al incremento de los gastos estatales que se deben hacer cuando ocurren los desastres y para la recuperación posterior.

En general, en el país la población no es consciente del beneficio de la prevención ante los riesgos de desastres, no existe control sobre el crecimiento urbano, no se ejerce la planificación urbana, la población toma las decisiones sobre la ubicación de sus viviendas, sin ejercicio riguroso por las autoridades de las normas de ubicación en lugares que no impliquen peligros potenciales. Eso conlleva a altos costos para la atención posterior de la reconstrucción sin reflexionar que es menos costosa la prevención ante los riesgos [7] y [9].

Ante la problemática expuesta buscamos la respuesta a cómo sería posible sincronizar la ayuda con las donaciones que recibe el Instituto Nacional de Defensa Civil (INDECI) del país y del exterior, cómo gestionar su entrega, y atender las necesidades prioritarias de los damnificados (sufren daño total) y afectados (sufren daño parcial). Además, se requiere determinar los lugares más seguros para refugiarlos, para almacenar y distribuir los suministros de ayuda, prestar la atención de salud, de alimentación, abrigo y otras necesidades básicas.

Se ha orientado el estudio a tres aspectos relevantes en la toma de decisiones relacionada con la cadena de suministro humanitaria, estos son:

a. Las decisiones sobre la gestión de los inventarios de kits de ayuda humanitaria y otros artículos individuales, útiles para brindar esta ayuda específicamente en el distrito de Chosica que sufre estos problemas con una frecuencia anual.

b. Se plantea un modelo sobre la localización de lugares seguros de refugio, almacenamiento y atención para casos de desastres en esta localidad. Este modelo aún no se ha evaluado cuantitativamente debido a que aún no se dispone de estudios geológicos que determinen las zonas seguras del distrito.

c. Se realiza un análisis de riesgo y vulnerabilidad haciendo uso de la información facilitada por el Instituto Geológico Minero y Metalúrgico (INGEMMET) y la Autoridad Nacional del Agua (ANA). Se realiza un estudio de las zonas del distrito donde no deberían localizarse viviendas y otras edificaciones por ser potencialmente lugares altamente vulnerables. 


\section{Metodología}

La investigación desarrollada es aplicada a una realidad concreta: el distrito de Chosica y su nivel es predictivo al orientarse a proponer soluciones ante escenarios posibles, anticipándose ante hechos potenciales. La fuente de datos es secundaria. La población está conformada por las zonas del distrito de Chosica afectadas con frecuencia por inundaciones. Se realiza un muestreo no aleatorio de las zonas afectadas. Se efectuó el análisis estadístico de los datos referentes a los diferentes aspectos estudiados en la investigación.

Se estimaron las distribuciones de probabilidad asociadas al proceso de simulación de sistemas de inventarios. Se desarrolló un proceso de simulación para determinar las políticas de reposición de inventarios de suministros de apoyo dado que los modelos clásicos de inventarios no son aplicables por sus caracter?sticas rígidas. Además, se desarrolló un modelo de localización de lugares seguros de centros de refugio-almacenamiento-atención y se realizó un análisis de riesgo y vulnerabilidad respecto al fenómeno de inundación.

Los datos e información fueron captados de documentos y entrevistas al personal especializado de INGEMMET y ANA, y de documentos publicados por INDECI e INGEMMET [8], [9] y [10].

\subsection{Logística Humanitaria}

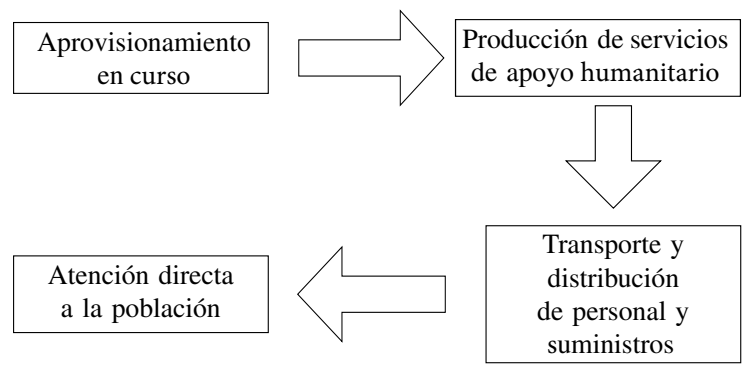

Figura 1. Cadena de Suministro

La Logística Humanitaria, se basa en el enfoque general de Logística [1], cuyo fin es que los productos lleguen a los consumidores en las condiciones requeridas, a través de una eficiente Cadena de Suministro [2], [15], sistema integrado por proveedores, productor, distribuidores, minoristas y consumidores finales, que interactúan en ese orden para satisfacer las necesidades del último eslabón de la cadena, el consumidor final.

La Logística Humanitaria comprende las acciones de refugio seguro y ayuda inmediata y permanente hasta que los damnificados y afectados, alcancen un nivel de vida aceptable e independiente respecto a la atención de sus necesidades básicas de alimentación, vestimenta, salud física y psicológica, refugio con servicios básicos esenciales y posteriormente reconstrucción y vivienda con esos servicios garantizados [4].

\subsection{Cadena de Suministro Humanitaria}

Es el sistema eslabonado de subsistemas que en el Perú es el Sistema Nacional de Defensa Civil(SINADECI), a cargo de INDECI, conformado por el subsistema de aprovisionamiento previo y en curso de subsistencias para el caso de desastres, el que provee de elementos necesarios demandados por los consumidores finales que son los damnificados y afectados, tales elementos son adquisiciones propias, donaciones internas y donaciones de otros países.

Existe además el subsistema productor de servicios de apoyo humanitario, el subsistema que realiza el transporte y distribución de suministros y de personal a los centros de refugio y el subsistema de refugiosalmacenes-centros de atención directa de damnificados y afectados donde se entregan los suministros, 
se brinda atención de salud y se atiende temporalmente las necesidades básicas. Este último subsistema requiere reforzarse en el caso tratado debido a que no hay certeza sobre la seguridad de su ubicación.

\subsection{Fuentes de Ayuda Humanitaria}

Cuando ocurren desastres de gran envergadura, la comunidad internacional ofrece y coordina la ayuda que puede prestar con la que el país afectado necesita. Esa ayuda generalmente llega oportunamente y de acuerdo a lo requerido, aunque existen casos excepcionales de ayuda en mal estado o ayuda innecesaria que el gobierno debe recibir y hacerse cargo de los gastos de desembarque.

Respecto a la ayuda de fuentes internas suele producirse un conflicto, una situación de menor control. Con frecuencia, la población dona suministros variados sin conocer los reales déficits de cada artículo en los almacenes oficiales o sin conocer las reales necesidades, desarrollándose iniciativas de organizaciones privadas [7].

Con los elementos donados se preparan paquetes con diversos artículos esenciales, agrupados en kits de higiene, kits de alimentos, kits familiares y también se entregan artículos individuales [10]. Se produce exceso de ciertas ayudas y escasez de otras, exigiéndose a las instituciones oficiales una reacción rápida respecto al gerenciamiento de la ayuda, a la comunicación, a la sensibilización de la población sobre la ayuda realmente necesaria, sobre los centros de acopio y de distribución. Ello indica que se requiere una planificación previa o estimada y una organización precisa y adecuada luego de la ocurrencia del desastre para evitar esfuerzos redundantes e innecesarios. Esto optimizaría la eficiencia del proceso. El proceso de ayuda humanitaria es representable como:
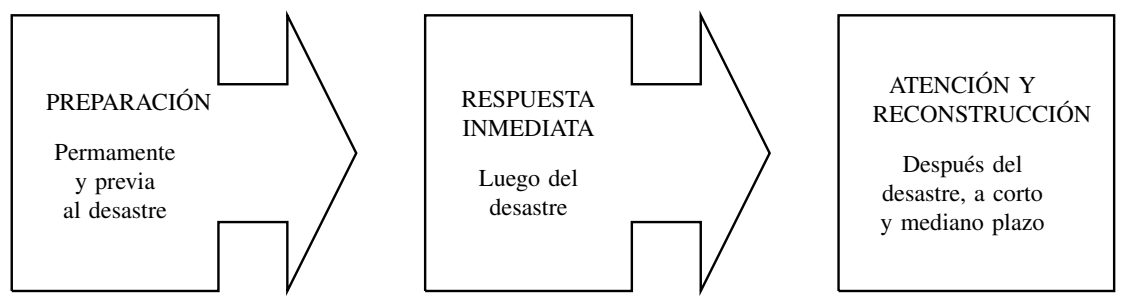

Figura 2. Proceso de ayuda humanitaria

En cada etapa de este proceso, la Logística Humanitaria cumple un rol esencial, la Preparación es una etapa estratégica y permanente en la que se produce el abastecimiento y almacenamiento de suministros no perecibles de acuerdo a la demanda estimada, esos artículos son compartibles para diferentes tipos de desastres, naturales o no: inundaciones, terremotos, tsunamis, incendios, heladas, huaycos, los cuales se espera que no ocurran simultáneamente en cuyo caso la demanda se multiplicaría.

La Respuesta Inmediata significa obtener o recibir la información de forma inmediata de las zonas afectadas por el fenómeno y enviar personal y suministros con inmediatez. La Atención y Reconstrucción se brindan desde el momento que ocurre el desastre durante el mediano y largo plazo hasta que se ha logrado la recuperación de las condiciones de supervivencia e independencia.

\subsection{Inventarios}

Desde el punto de vista de la Investigación de Operaciones, se requiere mantener en situaciones de desastres, inventarios de elementos de diferente naturaleza, es decir se requiere el almacenamiento o el registro de la disponibilidad de determinados elementos, así tenemos, en el caso de la Logística Humanitaria, inventarios de: recursos humanos como rescatistas, personal médico, paramédico y voluntarios.; inventarios de suministros no perecibles en corto plazo como los elementos de ayuda de los cuales se debe mantener un registro, codificación, catalogación, cuantificación y ubicación para facilitar su disponibilidad y traslado luego del desastre.; inventario de centros seguros de refugio-almacenamiento-atención, así 
como su capacidad, servicios disponibles y servicios potenciales [7] y [9].

De acuerdo al estudio desarrollado, los elementos asociados a estos tres tipos de inventario se gestionan con eficiencia a través de procesos de simulación estocástica útil debido a su mayor flexibilidad frente a los modelos clásicos de inventarios. Estos modelos requieren la estimación de distribuciones de probabilidad en la cual se utilizan métodos estadísticos.

\section{Decisiones en los sistemas de inventarios}

En un sistema de inventarios, existen dos tipos de decisiones fundamentales asociadas con la disponibilidad de artículos en inventarios, que expresadas como preguntas son: ¿cuánto pedir de cada artículo?, es decir, de qué cantidad de un artículo debe reabastecerse el inventario para tener la cantidad suficiente para responder a la demanda en cualquier momento del tiempo.

La otra pregunta es ¿cuándo pedir de cada artículo?, es decir, con qué frecuencia debe reabastecerse el inventario considerando que debido a la demanda del artículo su cantidad disminuye a lo largo del tiempo. Debe considerarse que ese reabastecimiento por lo general no es inmediato, ocurre después de un plazo dado desde el momento que se hace el pedido al proveedor hasta el momento que llega ese pedido al almacén.

\section{Inventarios en la cadena de suministro humanitaria}

Cuando se dispone de un registro histórico de la ocurrencia de desastres en un lugar determinado, es posible determinar la probabilidad de ocurrencia, asociada a la estacionalidad y recurrencia. Nuestra propuesta consiste en desarrollar el siguiente proceso:

a. Determinar la máxima demanda histórica por suministro, entre todos los tipos de eventos de desastres naturales ocurridos en esa localidad o zona.

b. Mantener inventarios de suministros, organizados o no en kits, necesarios para atender los diferentes tipos de desastres tomando como referencia la probabilidad de ocurrencia de cada tipo de desastre.

c. Gestionar la reposición de inventarios de acuerdo a los siguientes aspectos:

- En el caso de alimentos envasados, medicinas y sangre, retirar de los inventarios las unidades con fechas próximas a vencer y colocar una orden para pronta sustitución.

- Restituir en el más breve plazo tanto los faltantes de los artículos indicados como otros necesarios para la asistencia humanitaria.

- Aprovechar las economías de escala como los descuentos por cantidad comprada, a partir de los almacenes nacionales, a fin de minimizar los costos de reposición de inventarios.

\section{Modelo a utilizar para reaprovisionar inventarios en la cadena de suministro humanitaria}

Para el período entre desastres, se propone usar un modelo aproximado de revisión continua, que puede ser diaria, para determinar la vigencia y el estado, especialmente de los alimentos almacenados, no perecibles en corto tiempo, como los envasados. También se puede aplicar este método en el caso de inventarios de suministros que pueden sufrir deterioro u obsolescencia. INDECI [10], recomienda adquirir artículos cuya caducidad mínima sea de un año.

Si se tiene la cantidad suficiente y máxima posible con fecha vigente, no es necesario tomar decisiones de reposición, solo se hará cuando exista caducidad, en cuyo caso se revisará el registro de productos almacenados y se repondrá los artículos caducos.

Si todos los artículos tienen vigencia pero no se dispone de la cantidad máxima posible, se buscará incrementar el nivel del inventario colocando órdenes de reaprovisionamiento para recuperar el nivel máximo. 
El modelo que se propone, debe permitir actualizar siempre el nivel del inventario hasta una cantidad máxima a la que denominaremos QMAX, de tal manera que cuando observemos cualquiera de estos aspectos: la caducidad de los elementos del inventario o el estar el nivel del inventario por debajo del máximo, se tomarán las medidas para actualizar el nivel al máximo necesario, QMAX, para atender desastres, sin riesgo de escasez. Entonces, planteamos:

Si el inventario del $i$-ésimo kit o artículo está en el nivel $Q M A X_{i}$, reabastecer inmediatamente el inventario hasta alcanzar el nivel $Q M A X_{i}$.

Si existen unidades del $j$-ésimo artículo que han caducado, retirar las unidades caducas, disminuirlas de $Q M A X_{j}$ y reabastecer de inmediato el inventario hasta alcanzar el nivel $Q M A X_{j}$.

Así, la política de inventarios tiene a QMAX como el punto referencial y permanente de reabastecimiento para cada artículo o Kit. Ver la tabla 1 sobre kits y artículos individuales, que distribuye INDECI en casos de desastres.

Para el distrito de Chosica, consideramos que hay artículos individuales que tienen características similares respecto a la demanda que podemos agruparlos en dos categorías siendo la tercera categoría conformada por los kits.

Se estimó, para un evento crítico, una distribución de probabilidad uniforme de ocurrencia de una inundación que damnifique y afecte entre el $5 \%$ y $10 \%$ de la población del distrito, es decir, a un máximo de 30 mil de sus aproximadamente 300 mil habitantes, siendo así 30 mil el valor máximo referencial.

Proponiendo que debería tenerse en los almacenes distritales una capacidad de atención inmediata del $10 \%$ del total requerido y el resto desde el almacén nacional ubicado en Lima, se simuló un sistema de inventario de revisión diaria para el caso de desastres el cual al implantarse estaría a cargo del SIGAOSistema Integral para la gestión administrativa y de operaciones de INDECI. 
Tabla 1. Kits y artículos que distribuye INDECI en casos de desastres

\begin{tabular}{|c|c|}
\hline FAMILIA DAMNIFICADA & PERSONA DAMNIFICADA \\
\hline Artículos por familia & Artículos por persona \\
\hline 1 módulo o carpa & 1 cama o colchón \\
\hline $\begin{array}{l}15 \text { planchas de calamina, si no se consi- } \\
\text { dera módulo }\end{array}$ & 1 frazada o colcha \\
\hline 10 planchas de triplay & $\begin{array}{l}1 \text { mosquitero (selva o sierra-supeditado } \\
\text { al fenómeno)* }\end{array}$ \\
\hline 10 metros de plástico & 1 kit de utensilios. Plato, vaso, cuchara \\
\hline 1 bidón Sansón & 1 ración fría de alimento por día \\
\hline 1 balde & \\
\hline $\begin{array}{l}0.20 \text { de fardo de Ropa x persona (1 far- } \\
\text { do }=20 \mathrm{Kg})\end{array}$ & \\
\hline 1 Cucharón & \\
\hline 1 Cuchillo & \\
\hline FAMILIA AFECTADA & PERSONA AFECTADA \\
\hline Artículos que se entrega por familia & Artículos que se entrega por persona \\
\hline $\begin{array}{l}05 \text { m. de plástico (si no se considera mó- } \\
\text { dulo) }\end{array}$ & 01 Ración fría de alimento \\
\hline 0.20 de fardo de Ropa x persona & $\begin{array}{l}01 \text { Frazada o Colcha (supeditado al } \\
\text { fenómeno) }\end{array}$ \\
\hline $\begin{array}{l}\text { Registrar otras necesidades si la evalua- } \\
\text { ción de daños lo amerita }\end{array}$ & $\begin{array}{l}01 \text { Mosquitero (selva o sierra - supedi- } \\
\text { tado al fenómeno)* }\end{array}$ \\
\hline & Kit de utensilios (Plato. Vaso. Cuchara) \\
\hline & $\begin{array}{l}\text { Registrar otras necesidades si la evalua- } \\
\text { ción de daños lo amerita }\end{array}$ \\
\hline *El estudio sugiere sea para cualquier reg & \\
\hline \multicolumn{2}{|c|}{ TRABAJOS COMUNALES } \\
\hline $\begin{array}{c}\text { Equipos } \\
\text { (Por Comunidad - } 50 \text { Familias) }\end{array}$ & $\begin{array}{c}\text { Herramientas } \\
\text { (Cuadrilla de } 10 \text { personas) }\end{array}$ \\
\hline $\begin{array}{l}1 \text { Un Grupo Electrógeno } \\
1 \text { Motobomba con accesorios }\end{array}$ & $\begin{array}{l}5 \text { Palas } \\
5 \text { Picos }\end{array}$ \\
\hline \multicolumn{2}{|c|}{ TRABAJOS COMUNALES } \\
\hline $\begin{array}{c}\text { Equipos } \\
\text { (Por Comunidad - } 50 \text { Familias) }\end{array}$ & $\begin{array}{c}\text { Herramientas } \\
\text { (Cuadrilla de } 10 \text { personas) }\end{array}$ \\
\hline 1 Equipo de Tratamiento de Agua & 2 Barretas \\
\hline $\begin{array}{l}\text { Registrar otras necesidades si la evalua- } \\
\text { ción de daños lo amerita }\end{array}$ & 1 Carretilla \\
\hline
\end{tabular}


Tabla 2. Resultados de la simulación según escenario

\begin{tabular}{|c|c|c|c|c|}
\hline \multirow{2}{*}{$\begin{array}{l}\text { Escenario } \\
\text { posible }\end{array}$} & \multirow{2}{*}{$\begin{array}{l}\text { Tipo de Ayuda } \\
\text { Humanitaria }\end{array}$} & \multicolumn{2}{|c|}{$\begin{array}{c}\text { Ayuda Humanitaria } \\
\text { proveniente del: }\end{array}$} & \multirow[b]{2}{*}{ Total } \\
\hline & & $\begin{array}{c}\text { Almacén de } \\
\text { Chosica } \\
10 \%\end{array}$ & $\begin{array}{c}\text { Almacén de } \\
\text { Nacional de Lima } \\
90 \%\end{array}$ & \\
\hline \multirow[t]{3}{*}{14000 damnificados } & Kits individuales & 1400 & 12600 & 14000 \\
\hline & Carpas & 234 & 2100 & 2334 \\
\hline & Kits familiares & 234 & 2100 & 2334 \\
\hline \multirow{3}{*}{$\begin{array}{l}7000 \text { damnificados } \\
7000 \text { afectados }\end{array}$} & Kits individuales & 1400 & 12600 & 14000 \\
\hline & Carpas & 117 & 1050 & 1167 \\
\hline & Kits familiares & 117 & 1050 & 1167 \\
\hline \multirow{3}{*}{$\begin{array}{l}7000 \text { damnificados } \\
7000 \text { afectados }\end{array}$} & Kits individuales & 1400 & 12600 & 14000 \\
\hline & Carpas & 70 & 630 & 700 \\
\hline & Kits familiares & 70 & 630 & 700 \\
\hline
\end{tabular}

De este modo puede simularse una cantidad diversa de escenarios posibles. Finalmente, evaluando la posibilidad de ocurrencia de los casos extremos, tenemos los resultados presentados en la tabla 3.

Tabla 3. Resultados para escenarios extremos

\begin{tabular}{|c|c|c|c|c|}
\hline \multirow{2}{*}{$\begin{array}{l}\text { Escenario } \\
\text { posible }\end{array}$} & \multirow{2}{*}{$\begin{array}{l}\text { Tipo de Ayuda } \\
\text { Humanitaria }\end{array}$} & \multicolumn{2}{|c|}{$\begin{array}{c}\text { Ayuda Humanitaria } \\
\text { proveniente del: }\end{array}$} & \multirow[b]{2}{*}{ Total } \\
\hline & & $\begin{array}{c}\text { Almacén de } \\
\text { Chosica } \\
10 \%\end{array}$ & $\begin{array}{c}\text { Almacén de } \\
\text { Nacional de Lima } \\
90 \%\end{array}$ & \\
\hline \multirow[t]{3}{*}{30000 damnificados } & Kits individuales & 3000 & 27000 & 30000 \\
\hline & Carpas & 500 & 4500 & 5000 \\
\hline & Kits familiares & 500 & 4500 & 5000 \\
\hline \multirow{3}{*}{$\begin{array}{l}22500 \text { damnificados } \\
7500 \text { afectados }\end{array}$} & Kits individuales & 3000 & 27000 & 30000 \\
\hline & Carpas & 375 & 3375 & 3750 \\
\hline & Kits familiares & 375 & 3375 & 3750 \\
\hline \multirow{3}{*}{$\begin{array}{l}15000 \text { damnificados } \\
15000 \text { afectados }\end{array}$} & Kits individuales & 3000 & 27000 & 30000 \\
\hline & Carpas & 250 & 2250 & 2500 \\
\hline & Kits familiares & 250 & 2250 & 2500 \\
\hline \multirow{3}{*}{$\begin{array}{l}7500 \text { damnificados } \\
22500 \text { afectados }\end{array}$} & Kits individuales & 3000 & 27000 & 30000 \\
\hline & Carpas & 125 & 1125 & 1250 \\
\hline & Kits familiares & 125 & 1125 & 1250 \\
\hline \multirow{3}{*}{$\begin{array}{l}5000 \text { damnificados } \\
25000 \text { afectados }\end{array}$} & Kits individuales & 3000 & 27000 & 30000 \\
\hline & Carpas & 84 & 750 & 834 \\
\hline & Kits familiares & 84 & 750 & 834 \\
\hline \multirow{3}{*}{$\begin{array}{l}2500 \text { damnificados } \\
28500 \text { afectados }\end{array}$} & Kits individuales & 3000 & 2700 & 30000 \\
\hline & Carpas & 42 & 375 & 417 \\
\hline & Kits familiares & 42 & 375 & 417 \\
\hline \multirow[t]{3}{*}{30000 afectados } & Kits individuales & 3000 & 27000 & 30000 \\
\hline & Carpas & - & - & - \\
\hline & Kits familiares & - & - & - \\
\hline
\end{tabular}




\subsection{Localización}

Un problema de localización es tal que, conocidos un conjunto de puntos-zonas posibles en las cuales podrían ubicarse determinados centros de producción, servicios, almacenes, viviendas, entre otros, se requiere elegir uno o más de ellos para situar uno o más de los centros mencionados, bajo criterios de minimizar algún factor como costo, tiempo, distancia total, por obtener suministros, almacenarlos y finalmente distribuirlos, es por ello que se suelen tratar bajo un enfoque de Cadena de Suministro.

Se trata de determinar el mejor lugar o los mejores lugares entre un conjunto de lugares posibles, para ubicar uno o más de los centros necesarios, siguiendo algún criterio como los costos, la cercanía a los usuarios interesados, la cercanía a los proveedores de artículos, evaluados a través de la minimización de los costos, maximización de beneficios, minimización de tiempos, o para brindar servicios con oportunidad, se trata así de un problema de localización óptima.

\section{Localización de lugares de refugio-almacenamiento-atención}

En el caso de desastres, el problema se refiere a la falta de centros seguros de refugio, almacenamiento y atención, de localización permanente, adecuadamente ubicados, cercanos a concentraciones de la población, a fin de que acuda a ellos en el menor tiempo posible, inmediatamente después de ocurrido el desastre, en las diferentes localidades del país Serpa, [14]. Además, es también inherente al problema que no se dispone permanentemente de personal y otros recursos que deben asignarse para brindar atención en esos lugares de manera eficiente y eficaz, [3].

Un enfoque es tratar el problema mediante la Teoría de Grafos, para lo cual se requiere establecer los diferentes puntos seguros geográficamente y geológicamente, en los cuales es posible ubicar locales de refugio de poblaciones afectadas de un distrito, provincia o región. Cada punto seguro es identificado como una unidad o nodo. Entre un par de nodos puede existir o no una relación que para el caso de desastres tales como las inundaciones es la "posibilidad de acceso mediante algún medio de transporte". Entonces, si existe tal relación entre un par de nodos es posible enviar elementos entre ellos.

Un grafo $G=(N, A)$ es una estructura conformada por un conjunto $N$ de nodos y un conjunto $A$ de arcos o aristas. Los arcos expresan relaciones orientadas entre nodos y las aristas representan relaciones sin orientación. Así, si $N=\{a, b, c, d, e, \ldots\}$ y si $A=\{(a, c),(a, e),(d, b), \ldots\}$, entonces, el par ordenado $(i, j)$ expresa que existe una relación orientada entre los nodos, cuya orientación va de $i$ hacia $j, \mathrm{y}$ $A=\{[c, b],[e, d],[a, a], \ldots\}$, indica que existe una relación no orientada entre los nodos. De este modo, para un problema asociado con desastres es conveniente adoptar una estructura de grafo no orientado que simbolice la comunicación en ambos sentidos entre pares de nodos que representan centros de refugioalmacenamiento-atención

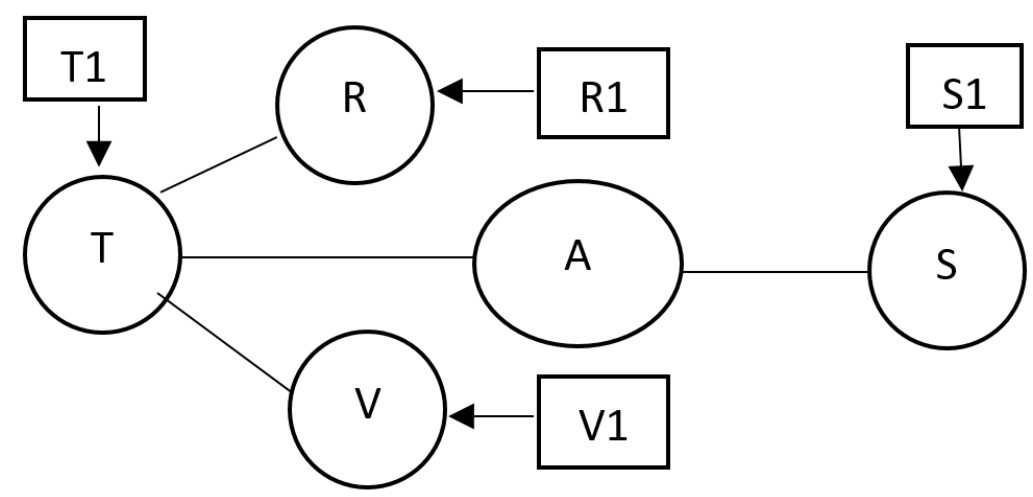

Figura 3. Grafo no orientado representando la comunicación entre los centros de refugio $\mathrm{R}, \mathrm{S}, \mathrm{T}, \mathrm{V}$ y el almacén nacional $\mathrm{G}$

En la figura 3 el nodo A representa el almacén nacional, los nodos R1, S1, T1, V1 representan a las 
poblaciones aledañas cercanas a los nodos correspondientes R, S, T, V, potencialmente asignables a tales nodos. A, representa el almacén central desde donde se envía ayuda a los almacenes del distrito, como puede observarse no es posible enviar en forma directa ayuda a todos los centros de atención pero sí es posible transbordarla desde centros intermedios, así por ejemplo, si es necesario enviar ayuda desde el almacén central al centro de refugio $\mathrm{R}$ debe enviarse a $\mathrm{T}$ y luego transbordarla a V. Como veremos posteriormente, los nodos circulares $\mathrm{R}, \mathrm{S}, \mathrm{T}, \mathrm{V}$ donde se ubican los centros de atención se identifican como medianas.

Los problemas de localización han sido ampliamente tratados por diversos autores, en particular en [12], referenciado en [3] se utiliza el enfoque de las p-medianas que se aplica en este estudio. Este problema se presenta como uno de p-medianas capacitado, el cual se representa mediante el siguiente modelo de programación lineal entera binaria.

El problema de las p medianas, consiste en que se tiene un conjunto de elementos o usuarios que requieren un servicio y que puede dividirse en p grupos. Cada grupo queda definido por los elementos que lo conforman y por la ubicación de la instalación donde se brinda el servicio, esa ubicación se conoce como mediana. Se trata de asignar a cada elemento a la mediana más cercana.

Visto como el problema de localización de centros de ayuda humanitaria, se tiene al conjunto estimado máximo de pobladores afectados y damnificados que requieren el servicio de atención luego de un desastre. Ese conjunto de pobladores puede dividirse en p grupos. Cada grupo queda definido por los pobladores que conforman el grupo y por la ubicación de la instalación más cercana al grupo, tal ubicación se identifica como la mediana asociada al grupo.

El modelo representativo es:

Minimizar $\sum_{i=1}^{n} \sum_{j=1}^{n} d_{i j} x_{i j}$

Sujeto a:

$\sum_{j=1}^{m} x_{i j}=1 ; i=1,2, \ldots, n$

$\sum_{j=1}^{m} y_{j}=p ; i \in \mathbb{N}$

$\sum_{i=1}^{n} x_{i j} \leq R_{j} y_{j} ; j=1,2, \ldots, m$

$x_{i j} \in\{0,1\}, i=1,2, \ldots, n ; j=1,2, \ldots, m$

Cada nodo $i$ representa una zona del distrito donde se encuentra un grupo de pobladores afectados y/o damnificados y cada nodo $j$ representa un lugar posible de localización de un centro de atención, o mediana.

La función objetivo expresada en (1) indica la minimización de la distancia total recorrida por todos los pobladores afectados y damnificados que necesitan ayuda.

La restricción (2) garantiza que cada poblador solo sea asignado a un centro de atención o mediana.

La restricción (3) indica que de todas las ubicaciones posibles solo se elegirán p, es decir, solo habrá p centros de atención o medianas.

La restricción (4) está asociada con la capacidad de cada mediana, así expresa que para cada mediana j se asignará como máximo Rj pobladores que la máxima capacidad del centro de atención o mediana j.

Así tenemos que:

$x_{i j}= \begin{cases}1 & \text { si el poblador } i \text { se asigna al centro de atención o mediana } j \\ 0 & \text { si no se asigna }\end{cases}$ 
$i=1,2, \ldots, n ; j=1,2, \ldots, m$

$y_{j}= \begin{cases}1 & \text { si se ubica el centro de atención o mediana en la localización } j \\ 0 & \text { si no se asigna }\end{cases}$

$d_{i j}$ : distancia a recorrer por el poblador afectado o damnificado $i$ asignado a la mediana $j$.

$R_{j}$ : capacidad del centro de atención o mediana $j$.

\subsection{Riesgo}

Definiremos los términos asociados al concepto de riesgo: peligro, desastre, vulnerabilidad y el propio término de riesgo. Se identifica como peligro, a un hecho asociado con la probabilidad de ocurrencia de un fenómeno natural, o no, que de ocurrir producirá daño a la fauna, flora, a las personas y sus pertenencias, afectando por tanto a su ambiente. "Los peligros, se caracterizan por su severidad, intensidad, magnitud y probabilidad de ocurrencia. Además, la magnitud del impacto depende principalmente de la exposición y vulnerabilidad del elemento afectado" (INDECI, 2017). Por ello es necesario identificar los peligros y sus características para evitarlos o minimizar su impacto. Un desastre es el resultado de un peligro que afecta severamente a la población. Los desastres pueden ser naturales ó tecnológicos cuando los produce el ser humano. Así tenemos tsunamis, terremotos, incendios, inundaciones, deslizamientos, huaycos, nevadas, friajes, explosiones, fugas de gas, entre otros, [9].

Según la Oficina de las Naciones Unidas para la reducción del riesgo de desastres, "vulnerabilidad es el conjunto de características en un sistema que lo hacen susceptible de sufrir los efectos de un peligro" [13]. La vulnerabilidad es física cuando por sus condiciones físicas un ambiente o una población corre riesgo de peligro y es económica cuando la población tiene dificultad para protegerse y recuperarse del peligro.

El riesgo se define como la probabilidad de que un peligro se convierta en un desastre, produciendo daños y pérdidas.

$$
\text { RIESGO }=\text { PELIGRO } \times \text { VULNERABILIDAD }
$$

El riesgo disminuye cuando se identifican los peligros y vulnerabilidades que enfrenta una persona o una población y se desarrollan actividades para eliminarlo o minimizarlo.

Respecto al problema de riesgo y vulnerabilidad en el distrito de Chosica, ocasionados por inundaciones, se ha recopilado información histórica sobre los riesgos a través del tiempo que han producido eventos de inundaciones por lluvias, desbordes del río Rímac y huaycos, de información proporcionada por INGEMMET y ANA [10]. Son 11 las quebradas de alto riesgo donde está asentada la población y existen 101 diques de los cuales aproximadamente 70 están dañados. Gran parte de la población está asentada en las laderas de los cerros, en las quebradas por donde antes se han deslizado huaycos. Las geomallas que se colocaron están colmatadas, no han sido limpiadas, por tanto, no soportarán nuevos deslizamientos de detritos.

Al inicio del año 2018, sin haberse producido Fenómeno El Niño, siete mil familias estuvieron en alto riesgo por el incremento del nivel de los ríos Rímac y Huaycoloro, [5]. A los diques dañados, se agrega la construcción de espigones particulares que desvían el cauce de las aguas en grandes volúmenes hacia zonas antes seguras convirtiéndolas en zonas de alto riesgo, además se realiza la extracción de arena y piedra de ambos ríos produciéndose cambios que generan grandes caídas de agua que llegan con fuerza a las zonas bajas produciendo inundaciones.

Las responsabilidades están compartidas: la población construye sus viviendas en zonas de alto riesgo como las quebradas y construye espigones que afectan al río en tanto que el municipio no hace respetar la zonificación para construcción de viviendas, incrementándose el riesgo.

El análisis de diversos documentos proporcionados por [10], sobre la evaluación de diferentes quebradas en Chosica ha permitido determinar las zonas de alto riesgo en las que no debe construirse, de las 
que debe retirarse la población y reubicarse en zonas de menor riesgo.

Otro aspecto importante es que Chosica tiene pocas zonas de bajo peligro, está rodeada de fuentes de peligro permanente. Entre sus puntos críticos se encuentran las quebradas Carossio, Libertad, Mariscal Castilla, Nicolás de Piérola, Santo Domingo y San Antonio de Pedregal, con peligro de deslizamientos y las zonas de La Perla, Malecón, Cañaverales, Campanillas, Huampaní, Ñaña, Carapongo y Huachipa, vulnerables a deslizamientos [10].

El estudio ha comprendido la recopilación de datos secundarios de una muestra no aleatoria de zonas de peligro hallándose las ubicaciones geográficas, las ubicaciones UTM y las coordenadas cartesianas de ubicaciones o puntos de riesgo. También se ha hallado la matriz de distancias entre puntos de riesgo que es de utilidad para el desplazamiento de ayuda entre puntos afectados por un desastre [10] y [14]. La información cuantitativa referente a riesgos se presenta en el Anexo.

\section{Discusión}

A partir de la información disponible sobre esta problemática del distrito de Chosica, verificamos que haciendo uso de herramientas de Estadística, modelos de simulación para determinar ante escenarios alternativos de impactos de inundaciones, es posible tomar decisiones sobre la ubicación de lugares seguros de refugio, almacenamiento y atención de pobladores.

Se muestra que la simulación es una herramienta útil para plantear diversos escenarios de impacto sobre la población y la respuesta sobre el abastecimiento de los almacenes de Chosica y del almacén nacional para abastecer de la ayuda. Como consecuencia se establece la capacidad de cada almacén del distrito.

También, considerando las características del problema se ha planteado un modelo de localización de centros de atención basados en información sobre todos los lugares seguros para la ubicación de tales instalaciones. Su solución optimiza el número de centros de atención a instalar en una localidad.

Los datos requeridos, que aún no cuentan las entidades oficiales, como INGEMMET y ANA son los de las medianas j, siendo los demás datos generados como consecuencia de estos. Sin embargo, la localización de al menos un centro en p zonas seguras que se identifiquen garantizará la atención con mayor prontitud que en la situación actual en que se cuenta con un único centro de refugio -almacenamiento - atención en el distrito.

\section{Conclusiones}

Es posible sincronizar los requerimientos de ayuda de las poblaciones afectadas con las disponibilidades en los almacenes, estableciendo almacenes o ampliando su capacidad de acuerdo a los requerimientos potenciales según registros históricos, lo cual contribuye a optimizar la atención a los damnificados y afectados.

La localización de centros de refugio-almacenamiento-atención en lugares seguros del distrito de Chosica, contribuirá en términos generales a acceder a la atención en forma inmediata o en tiempos menores que si se espera la ayuda desde otros distritos o desde el almacén nacional ubicado en la ciudad de Lima, esto en cuanto se disponga de los datos generados por los estudios de suelos y otros estudios técnicos, que se usarán para evaluar el modelo propuesto que está validado teóricamente.

Es posible mantener disponibilidades de bienes básicos de ayuda humanitaria suficientes para la atención de emergencias, tales como las inundaciones y otros fenómenos similares, en el distrito de Chosica, gestionando con eficiencia los inventarios de adquisiciones y donaciones y manteniéndolos abastecidos en su capacidad máxima a lo largo del tiempo. 


\section{Agradecimientos}

Agradecimiento especial a las instituciones INGEMMET y ANA por las facilidades brindadas para acceder a la información necesaria para esta investigación y por la alta disposición de sus funcionarios para facilitarla y ampliarla.

\section{Referencias bibliográficas}

[1] Afshar, A. Haggani (2012).Modeling integrated Supply Chain Logistics in Real-time Large-Scale Disaster Relief Operations. Socio-Economic Planning Sciences. Elsevier, Vol. 46(4), pages 327-338.

[2] Balcick, B.; Beamon, B.M.; Krejci, C.C.;Muramatsu, K. M. ; Ramírez, M. (2010) Coordination in Human Knowledge and Environmental Humanitarian Relief Chains: Practices, challenges and oportunities. International Journal of Production Economics, 126(1), 22-34.

[3] Berger, E.; Gambini, I.; Alcalde, J. (2009). Localización en redes y simulación de redes de Petri para la atención de poblaciones afectadas por desastres naturales. PESQUIMAT XII(1), 64-69.

[4] Carrasco, J. (2017). Aplicación de la Logística Humanitaria para atender la emergencia ocasionada por El Niño Costero en Piura. Tesis. Universidad de Piura.

[5] Presidencia del Consejo de Ministros. Normas Legales. (26 de mayo de 2011). Decreto Supremo que aprueba el Reglamento de la Ley 29664 que crea el Sistema Nacional de Gestión del Riesgo de Desastres (SINAGERD). Diario El Peruano. pp. 443206-443218.

[6] Editorial en portada. (25 de abril de 2017). La Reconstrucción después de los Desastres Naturales. Diario La República, pp. 2-3.

[7] Gígola, C. (2014). Logística Humanitaria. Aplicación de la Investigación de Operaciones a la atención de Desastres. Minicurso. XVII Congreso Latinoamericano de Investigación de Operaciones. México.

[8] INDECI-PNUD-CESAL (2013). Proyecto Escenarios de Riesgos de Desastres.

[9] INDECI-INEI-UNFPA (2015). Conversatorio sobre la Gestión del Riesgo de Desastres. Importancia de la Información Estadística.

[10] INDECI (2017). Plan Logístico INDECI AF-2017 para Ayuda Humanitaria ante Emergencia o Desastres. Informe No. 459-2016-INDECI.

[11] Núñez, S.; Sosa, N. y Lara, J. (2017). Diversos Informes de Opiniones Técnicas sobre las Quebradas de Chosica. INGEMMET. Lima.

[12] Pizzolato, N. (2004). Localización de Escuelas Públicas. Pesquisa Operacional,24(1), 11-131.

[13] Tomassini, R.; Van Wasenhove, L. (2009). Humanitarian Logistics. INSEAD.

[14] Serpa, V. (2014). Optimización y Localización de almacenes de abastecimiento para la atención de un terremoto de gran magnitud en Lima Metropolitana y Callao (tesis de pregrado). Pontificia Universidad Católica del Perú, Lima, Perú.

[15] Viera, O.; Moscatelli, S. y Tansini, L. (2012). Logística Humanitaria y su aplicación en Uruguay. Gerencia Tecnológica Informática, $11(30), 47-56$. 